\title{
Prevalence of Tuberculosis in Patients with Liver Cirrhosis
}

\author{
Dilip Sharma, ${ }^{1}$ Sudhamshu KC, ${ }^{1}$ Bikash Jaisi ${ }^{1}$ \\ ${ }^{1}$ Liver Unit, Department of Medicine, Bir Hospital, Kathmandu, Nepal.
}

\begin{abstract}
Background: Studies have shown that patients with liver cirrhosis are more susceptible to tuberculosis because of immune dysfunction; however there are only limited data. We carried out this study to know the prevalence and clinical characteristics of tuberculosis in patients with liver cirrhosis.

Methods: In this retrospective study, medical files of 200 consecutive patients admitted with diagnosis of liver cirrhosis in the medical ward of liver unit, Bir Hospital from January 2011 to December 2014 were evaluated for the presence of TB and clinical characteristics of these patients were analyzed.

Results: Eighteen patients were diagnosed to have tuberculosis with a prevalence of $9 \%$. The median age of patients with tuberculosis and liver cirrhosis was $53(35-71)$ years and $\mathrm{M}$ : F ratio was 2:1. 67\%, $\mathrm{P}=0.01)$. Median CTP score of patients were 8.5 (5-12) (CTP A: B: C: 3:7:8). Extra pulmonary tuberculosis was more common (67\%) than pulmonary (33\%). In extra pulmonary tuberculosis, distribution was as pleural effusion $(n=4)$, abdominal $(n=4)$, intestinal $(n=2)$, peritoneal $(n=9)$, and pott's spine $(n=2)$.

Conclusions: Tuberculosis is prevalent in patients with liver cirrhosis. Extra pulmonary tuberculosis was more compared to pulmonary tuberculosis. Tuberculosis was common in decompensated liver cirrhosis than compensated liver cirrhosis .

Keywords: Cirrhosis; extra pulmonary; pulmonary; tuberculosis.
\end{abstract}

\section{INTRODUCTION}

Despite good advancement in diagnosis and treatment, tuberculosis (TB) still remains a major threat to the world. ${ }^{1,2}$ Studies done on autoptic samples have shown a prevalence of $10 \%$ of Liver cirrhosis (LC) suggesting it as a common condition. ${ }^{3}$ Likewise, a higher prevalence of TB has been identified in patients with LC with a risk rise of 14-fold in cirrhotic patients with more so in decompensated disease. ${ }^{4}$ This is mainly due to immune dysfunction with associated higher virulence. ${ }^{5}$ In a study from Denmark, the incidence of TB was $168.6 / 100000$ in LC and those who acquired TB had a poorer prognosis. ${ }^{6}$ In similar studies, higher prevalence of TB in LC patients have been reported in India. In same study pulmonary TB has been identified as the commonest form. ${ }^{7}$ In another study conducted in India, extapulmonary TB has been suggested to be the commonest form in $80 \%$ to $90 \%$ of all the reported cases of TB. ${ }^{8}$ In patients with LC, extra- pulmonary cirrhosis was more common than pulmonary TB. ${ }^{9,10}$ In Nepal, tuberculosis still is a major public health issue with an estimated prevalence of 156 per 100,000 populations. ${ }^{11}$ The aim of this study the prevalence of tuberculosis in patients with liver cirrhosis.

\section{METHODS}

This is a retrospective study from Bir hospital, a tertiary referral center of Nepal. The medical files of diagnosed cases of liver cirrhosis of any etiology, consecutively admitted from January 2011 to December 2014 were reviewed to look for prevalence of tuberculosis of any sites. A total of 200 patients with liver cirrhosis were enrolled in this study. The basis of diagnosis of liver cirrhosis was clinical symptoms, laboratory parameters, imaging diagnosis and histopathology whenever available. The severity of the liver disease was assessed as per Child Pugh scoring system. 
The presenting complaints at admission were noted. The findings of general and systemic examinations like lymph node examination, ascites, pleural effusion, respiratory systems were meticulously reviewed and noted. In personal history, contact with patients of tuberculosis was particularly noted. Laboratory investigations, which remain the key for the diagnosis, were thoroughly reviewed and noted. Special attention was given to the body fluid examinations including ADA. All the chest $X$-rays were collected and findings were reviewed with pulmonologist. X-ray of limbs or spines were reviewed with radiologist. Ultrasound and $\mathrm{CT}$ scan of abdomen findings were also noted with emphasis of liver as well as findings of fluid in the pleural as well as peritoneal cavity. Endoscopy and colonoscopy findings were also reviewed and noted.

Those patients with incomplete investigations and doubtful diagnosis were excluded from the study despite the provisional diagnosis of tuberculosis infection. The final diagnosis was re-confirmed after detail discussion of the suspected patients. All the values were entered in excel file and SPSS version 20 was used for the statistical analysis using appropriate tools. Ethical approval was taken from the ethical review board of National Academy of Medical Sciences and consent was obtained from the medical record section of Bir hospital, NAMS.

\section{RESULTS}

A total of 238 consecutive medical records were reviewed and after exclusion required number of 200 patients data was available. The demographic profile of these patients is given in table 1 . There was male preponderance with a male: female ratio of 2.7:1 ( $M$ : F: 146: 54). The etiologies of liver cirrhosis are given in table 2. Alcohol was the main cause of liver cirrhosis followed by hepatitis B and C. Alcohol, hepatitis B and C accounted for $90 \%$ of the total patients. Based on child Pugh scoring, 38(19\%), 132(66\%) and $30(15 \%)$ were in A, $B$ and $C$ group, respectively.

Out of 200 cases, 18 cases were diagnosed to have TB at the time of discharge. Thus, the prevalence was $18 / 200$ which comes to $9 \%$. Extra pulmonary TB was more common in patients with cirrhosis (Pulmonary: Extra pulmonary: $33 \%: 67 \%, \mathrm{P}=0.01$ ). In extra pulmonary TB distribution was as pleural effusion, $n=4$, abdominal, $\mathrm{n}=4$, intestinal, $\mathrm{n}=2$, peritoneal, $\mathrm{n}=9$, pott's spine, $\mathrm{n}=2$. The median age of patients with tuberculosis and liver cirrhosis was 53 (35-71) years and $M: F$ ratio was 2:1. Median CTP score of patients were 8.5 (5-12) (CTP A: B: C: $3: 7: 8)$.

\section{Table 1. Demographic profile of the patients.}

\begin{tabular}{|c|c|c|}
\hline Age (Years) & Male (\%) & Female (\%) \\
\hline $30-40$ & $10(5 \%)$ & $2(1 \%)$ \\
\hline $41-50$ & $50(25 \%)$ & $20(10 \%)$ \\
\hline $51-60$ & $50(25 \%)$ & $18(9 \%)$ \\
\hline $61-70$ & $30(15 \%)$ & $12(6 \%)$ \\
\hline \multirow[t]{2}{*}{$>71$} & $6(3 \%)$ & $2(1 \%)$ \\
\hline & $146(73 \%)$ & $54(27 \%)$ \\
\hline Iaple 2. Etiology от live & rrnosis. & No (\%) \\
\hline Alcohol & & $102(51)$ \\
\hline Alcohol + hepatitis B & & $34(17)$ \\
\hline Alcohol + hepatitis C & & $16(8)$ \\
\hline Hepatitis B & & $20(10)$ \\
\hline Hepatitis C & & $8(4)$ \\
\hline Budd- Chiari syndrome & & $4(2)$ \\
\hline Autoimmune hepatitis & & $6(3)$ \\
\hline Primary Biliary cirrhosis & & $4(2)$ \\
\hline Wilson's disease & & $2(1)$ \\
\hline Cryptogenic & & $4(2)$ \\
\hline \multicolumn{3}{|c|}{$\begin{array}{l}\text { Table 3. Age distribution of patients with } \\
\text { tuberculosis and liver cirrhosis. }\end{array}$} \\
\hline Age (Years) & Male & Female \\
\hline $30-40$ & 1 & 0 \\
\hline $41-50$ & 2 & 1 \\
\hline $51-60$ & 7 & 4 \\
\hline $61-70$ & 2 & 1 \\
\hline$>71$ & 0 & 0 \\
\hline
\end{tabular}

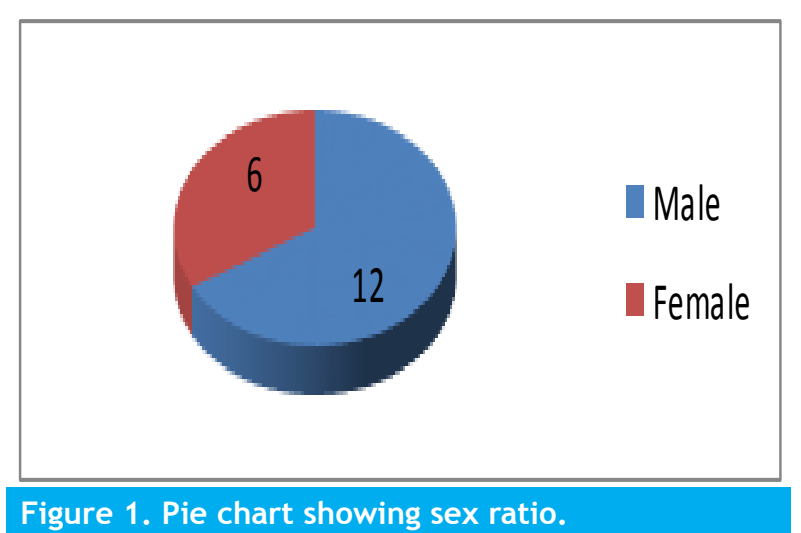




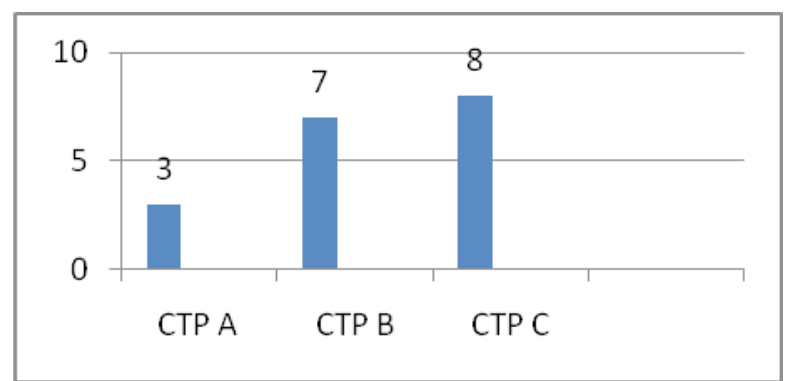

Figure 2. Bar diagram showing CTP score.

\section{DISCUSSION}

In the present study, we analysed the medical records of patients to investigate the prevalence of tuberculosis in patients with liver cirrhosis. We found the prevalence of TB in liver cirrhosis was $9 \%$, which was higher than the general population. Our results are consistent with previous studies which have shown a higher prevalence of TB in patients with LC when compared to general population. ${ }^{3,4,5}$

Similar to our findings, Baijal et al. have shown the prevalence rate of TB in cirrhotics which was 15 times higher than the general population. ${ }^{8}$

Previous studies have suggested Cirrhosis as a risk factor for extrapulmonary TB. In our study, the extra-pulmonary involvement in LC was more than the general population. Similar to our findings, Cho et al have reported $31 \%$ of the patients with liver cirrhosis had extrapulmonary TB with predominance of peritoneal TB. ${ }^{5}$ In contrast to our findings, Saigal et al have shown pulmonary tuberculosis as the commonest form. However, the prevalence of TB in LC patients was five times higher than the general population. $^{7}$

There is little information regarding the immunopathogenesis of TB in patients with LC. It is said that in patients with liver cirrhosis there is diminished clearance capacity of the reticloendothelial system. However, the condition to develop extrapulmonary TB in patients with LC due to immune dysfunction is still unclear. ${ }^{2}$ The diagnosis of extrapulmonary tuberculosis is challenging. Tubercular peritonitis most often mimics spontaneous bacterial peritonitis. The former occurs in less-advanced cirrhosis and ascitic fluid analysis show higher amount of mononuclear cells, protein and adenosine deaminase. However, the white blood cell count is lowered. ${ }^{16} \mathrm{In}$ a study conducted by Hillebrand et al more than $50 \%$ of tubercular peritonitis patients had cirrhosis mostly associated with alcohol. ${ }^{17}$

The pateints with liver cirrhosis usually demonstrate inpaired delayed type of hypersensitivity reaction so there is more chances of getting false negative tuberculin tests. In case of abdominal tuberculosis the AFB smears are generally negative. ${ }^{2}$

Previous studies showed tuberculosis was more in decompensated liver cirrhosis than compensated which is similar to our findings where majority of patients were child Pugh B and C. There was no statistical difference with the incidence of tuberculosis in liver cirrhosis patients according to the etiology.

\section{CONCLUSIONS}

Our study showed that prevalence of tuberculosis in cirrhotic patients is higher than general population. Patients with liver cirrhosis who develop tuberculosis are generally decompensated, the majority having Child- Turcotte -Pugh (CTP) grade B or C. Patients with liver cirrhosis have more frequent extra pulmonary involvement than general population.

\section{REFERENCES}

1. Williams C. Global tuberculosis control: WHO report 2011

2. Kumar N, Kedarisetty CK, Kumar S, Khillan V, Sarin SK. Antitubercular therapy in patients with cirrhosis: challenges and options. World J Gastroenterol. 2014;20(19):5760. [Full Text]

3. Tiribelli C, Croce LS, Polo S, Sodde M, Stanta G. Incidence of hepatocellular carcinoma in Italy: what could we learn from autoptic studies? Ital J Gastroenterol. 1991; 23: 448451.[Full Text]

4. Ramappa V, Aithal GP. Hepatotoxicity related to antituberculosis drugs: mechanisms and management. J Clin Exp Hepatol. 2013 Mar 31; 3(1):37-49.[Science Direct]

5. Choy J, Lee SM, Yoo CG, Kim YW, Han SK, Shim YS, Yim JJ. Clinical characteristics of tuberculosis in patients with liver cirrhosis. Respirology. 2007 May 1;12(3):401-5.[Full Text]

6. Thulstrup AM, Mølle I, Svendsen N, Sørensen HT. Incidence and prognosis of tuberculosis in patients with cirrhosis of the liver. A Danish nationwide population based study. Epidemiol Infect. 2000;124(2):221-5.[Full Text]

7. Saigal S, Nandeesh HP, Agarwal SR, Misra A, Jain SK, Sarin SK. High prevalence and profile of tuberculosis in chronic liver disease patients. Gastroenterology. 1998; 114:A38. 
8. Baijal R, Praveenkumar HR, Amarapurkar DN, Nagaraj K, Jain M. Prevalence of tuberculosis in patients with cirrhosis of liver in western India. Trop Doct. 2010; 40(3):163-4. [Full Text]

9. Mehta JB, Dutt A, Harvill L, Mathews KM. Epidemiology of extra pulmonary tuberculosis: a comparative analysis with pre-AIDS era. Chest. 1991;99(5):1134-8. [Science Direct]

10. Gonzalez OY, Adams G, Teeter LD, Bui TT, Musser JM, Graviss EA. Extra-pulmonary manifestations in a large metropolitan area with a low incidence of tuberculosis. The International Journal of Tuberculosis and Lung Disease. 2003; 7(12):1178-85.[Full Text]

11. Estimates of TB and MDR-TB burden are produced by WHO in consultation with countries. WHO GLOBAL REPORT; 2017-05-16.

12. Iglesias M, Macías MA, Correro F, Soria MJ. Miliary tuberculosis and cirrhosis. Revistaclínicaespañola. 1994;194(1):68.[Full Text]
13. 20th European Congress of clinical microbiology tuberculosis complicated with chronic liver and infectious disease, Vienna, Austria, 2010; 10-13.

14. Dhingra VK. Treatment of Tuberculosis in Liver Disease (A Clinician's view).

15. Harada H, Murai S, kojima H, et al. Diagnosis and treatment of Pulmonary disease. Niho Rinsho 1998; 56:321-6.

16. Kim NJ, Choo EJ, Kwak YG, Lee SO, Choi SH, Woo JH, Kim YS. Tuberculous peritonitis in cirrhotic patients: comparison of spontaneous bacterial peritonitis caused by Escherichia coli with tuberculous peritonitis. Scand J Infect Dis. 2009; 41: 852-856 [Full Text]

17. Hillebrand DJ, Runyon BA, Yasmineh WG, Rynders GP. Ascitic fluid adenosine deaminase insensitivity in detecting tuberculous peritonitis in the United States. Hepatology. 1996; 24: 1408-12 [Full Text] 